\title{
Of very young mice and men
} In two separate efforts, researchers isolate
pluripotent stem cells from the epiblast of
the post-implantation mouse embryo.

These are exciting times in the study of mammalian development. "Pluripotency is not something you come across every day," says Ron McKay at the US National Institutes of Health. Two recent papers, one from McKay's group with Richard Gardner at Oxford University (Tesar et al., 2007), and the other from Roger Pedersen's group at Cambridge University (Brons et al., 2007), now report the derivation of pluripotent cell lines from the post-implantation mouse epiblast.

Previous work indicated that mouse and human embryonic stem cells (ESCs) are different, in particular in their requirements for maintenance in culture. Researchers from both the Pedersen and McKay groups thus went back to the mouse embryo to identify a cell that is more similar to the human ESC.

Both groups could culture pluripotent cells from epiblasts of post-implantation mouse embryos and could maintain them under conditions that support the growth of human but not of mouse ESCs. Notably, both groups showed by analysis of global gene expression that epiblast stem cells and ESCs from mouse sort into different categories (Fig. 1), and that the gene expression profile of epi-cell lines is close to that of post-implantation epiblasts. This strongly indicates that the embryonic origin of the new mouse epiblast lines is indeed the post-implantation epiblast.

A major implication of this work is that human ESC lines are likely also to have their origin in the epiblast. "The human ESC and the new mouse cells both benefit from JAK inhibition, whereas it is lethal for proliferation of classic mouse ESCs," says McKay, "In addition, if you look at gene expression, the human ESC is similar to the mouse epi-cell and there's a whole set of genes that is missing from both, but which are clearly on in mouse ESCs." Further, chromatin immunoprecipitation (ChIP)-on-chip analysis of Oct3/4 targets suggests that mouse epi-cells
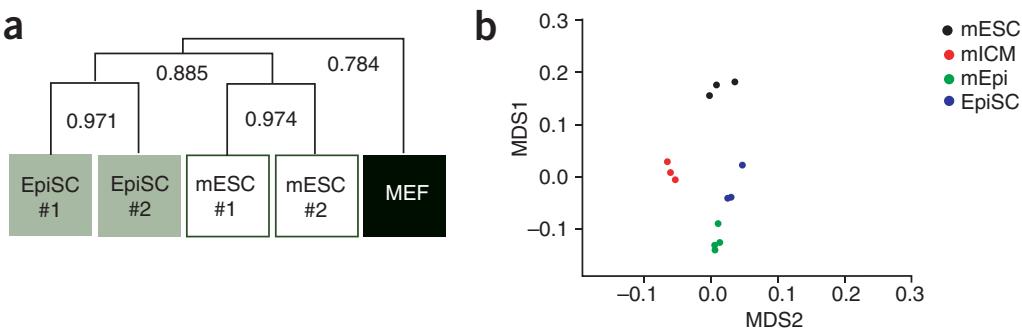

Figure 1 | Identity of mouse epiblast stem cells. (a,b) Global gene expression analysis shows that mouse epiblast stem cells are distinct from $\operatorname{ESCs}(\mathbf{a}, \mathbf{b})$ and that their profile is similar to that of primary mouse epiblasts (b). Reprinted from Nature.

are more similar to human ESCs than mouse ESCs are.

But there remains a significant nonoverlapping set of Oct3/4 targets between human ESCs and mouse epi-cells. "I don't think anybody knows for certain how close these cells are," says McKay, "but it is not a trivial similarity. It's reasonable to conclude that in humans, a species without diapause, even though ESCs are derived from the preimplantation embryo, the stable state is the epiblast state so those are the cells that you're going to grow." Comparative molecular studies of these different types of stem cell lines will contribute further to a complete understanding of their identity, suggests Ludovic Vallier, senior author on the other paper reporting the derivation of epiblast lines. "But I think we have to expect some differences," he predicts.

Although epiblast stem cell lines are demonstrably pluripotent in vitro, the cells do not generate chimeras when injected into blastocysts and implanted in utero. "There is a problem of incompatibility between the cells," says Vallier. "The epiblast cells simply don't colonize the blastocyst." Moreover, McKay points out that aggregation of the epi-cells with mouse morulae results in slowed development, suggesting that the epi-cells create an environment that is actively inhibitory for morula growth. One possible explanation for the incompatibility, favored by Vallier, lies in the developmental asynchrony between the blastocyst and the post-implantation epiblast stem cells. To test this idea, the scientists are trying to inject the epi-cells into later-stage embryos, to see if they can contribute to chimeras in that context.

"This work is going to be important for the derivation of stem cells from other mammalian species," says Vallier. Indeed, Vallier and colleagues use post-implantation epiblasts to derive pluripotent stem cell lines from the rat, which has not been possible so far. "People have been trying for a long time to get a source of pluripotent cells from pig or cow, and have been unsuccessful," he says. "If those cells are to be useful for any applications other than basic research, it will be important to solve the chimerism issue."

But perhaps most critically, the new epiblast stem cells will be a powerful addition to the existing in vitro models for studying cellfate specification in the early mammalian embryo. "The remarkable thing," as McKay puts it, "is these cells are isolated from the embryo at day 5.5 , but by 7.5 , in some sense it's all over. It doesn't look like an animal yet, but all the cell types are there. And how that happens is the big issue, really."

\section{Natalie de Souza}

\section{RESEARCH PAPERS}

Brons, I. et al. Derivation of pluripotent epiblast stem cells from mammalian embryos. Nature; published online 27 June 2007.

Tesar, P. et al. New cell lines from mouse epiblast share defining features with human embryonic stem cells. Nature; published online 27 June 2007. 